\title{
ON SURFACES OF FINITE TYPE IN EUCLIDEAN 3-SPACE
}

\author{
By Franki Dillen ${ }^{1)}$, Johan Pas ${ }^{1)}$ and Leopold Verstraelen
}

\begin{abstract}
We prove an extension of T. Takahashi's result on minimal submanifolds in Euclidean spaces and in spheres, and as a corollary obtain support for B. Y. Chen's conjecture which claims that the round spheres are the only compact surfaces of finite type in Euclidean 3-space.
\end{abstract}

Let $M^{n}$ be a (connected) $n$-dimensional submanifold in $\boldsymbol{E}^{m}$, the $m$-dimensional Euclidean space. Let $x, H$ and $\Delta$ respectively be the position vector field, the mean curvature field and the Laplace operator of the induced metric on $M^{n}$. Then, as is well known (see e.g. [2]),

$$
\Delta x=-n H \text {, }
$$

which shows, in particular, that $M^{n}$ is a minimal submanifold in $\boldsymbol{E}^{m}$ if and only if its coordinate functions are harmonic (i. e. they are eigenfunctions of $\Delta$ with eigenvalue 0 ). Moreover, in this context, T. Takahashi [6] proved that the submanifolds $M^{n}$ for which

$$
\Delta x=\lambda x,
$$

i.e. for which all coordinate functions are eigenfunctions of $\Delta$ with the same eigenvalue $\lambda \in \boldsymbol{R}$, are precisely either the minimal submanifolds of $\boldsymbol{E}^{m}(\lambda=0)$ or the minimal submanifolds $M^{n}$ of hyperspheres $S^{m-1}$ in $\boldsymbol{E}^{m}$ (the case when $\lambda \neq 0$, actually $\lambda>0$ ). In terms of B. Y. Chen's theory of submanifolds in $\boldsymbol{E}^{m}$ of finite type, condition (1.2) asserts that $M^{n}$ is of 1-type in $\boldsymbol{E}^{m}$. In general, a submanifold $M^{n}$ in $\boldsymbol{E}^{m}$ is said to be of finite type if its spectral decomposition of $x$ is finite, i.e. if

$$
x=x_{0}+\sum_{t=p}^{q} x_{t}
$$

where $p$ and $q$ are natural numbers, such that $x_{0} \in \boldsymbol{R}^{m}$ is a fixed vector and

$$
\Delta x_{t}=\lambda_{t} x_{t}
$$

\footnotetext{
1) Research Assistant of the National Fund of Scientific Research (Belgium)

Received March 3, 1989
} 
where $\lambda_{t}$ denotes an eigenvalue of $\Delta[\mathbf{1}][\mathbf{2}]$; when there are exactly $k$ nonconstant eigenvectors $x_{t}$ appearing in (1.3), which all belong to different eigenvalues $\lambda_{t}$, then $M^{n}$ is said to be of $k$-type in $\boldsymbol{E}^{m}$. Many important submanifolds in Euclidean spaces turn out to be of finite type in this sense. To find out whether or not a compact submanifold $M^{n}$ in $\boldsymbol{E}^{m}$ is of finite type, the following result is very useful.

THEOREM A. (B. Y. Chen [2])

(i) $M^{n}$ is of finite type in $\boldsymbol{E}^{m}$ if and only if there exists a non-trivial polynomial $Q$ (of one variable) such that $Q(\Delta) H=0$.

(ii) If $M^{n}$ is of finite type, then there exists a unique monic polynomial $P$ (of one variable), of least degree and such that $P(\Delta) H=0$.

(iii) If $M^{n}$ is of finite type, then $M^{n}$ is of $k$-type if and only if degree $P=k$.

The same results hold if $H$ is replaced by $x-x_{0}, x_{0}$ being the center of mass of $M^{n}$ in $E^{m}$.

In $[3]$, B. Y. Chen studies the following problem.

QUESTION. Other than minimal surfaces and ordinary spheres, which surfaces in $\boldsymbol{E}^{3}$ are of finite type?

Restricting attention to surfaces in $\boldsymbol{E}^{3}$, the above result on $\Delta x=\lambda x, \lambda \in \boldsymbol{R}$, can be stated as follows (which also somewhat clarifies the previous Question).

Theorem B. (T. Takahashi [6])

$A$ surface in $\boldsymbol{E}^{3}$ is of 1-type if and only of it is a sphere or a minımal surface.

With respect to the Question, the following result is quite interesting.

Theorem C. (B. Y. Chen [3])

A tube in $\boldsymbol{E}^{3}$ is of finite type of and only if it is a circular cylinder (which actually is of 2-type).

As a corollary we mention the following,

COROLlaRY D. (B. Y. Chen [3])

Every closed tube in $\boldsymbol{E}^{3}$ is of infinite type,

Which offers a partial solution to the following

CONJECTURE OF B. Y. ChEN.

Ordinary spheres are the only compact finite type surfaces in $\boldsymbol{E}^{3}$.

Of course, since there are no compact minimal surfaces $\boldsymbol{E}^{3}$, Theorem B settles the matter for 1-type surfaces.

In [5], O. Garay studies the hypersurfaces $M^{n}$ in $\boldsymbol{E}^{n+1}$ for which 


$$
\Delta x=A x,
$$

where $A$ is a diagonal matrix

$$
A=\left(\begin{array}{ccccc}
\lambda_{1} & & & & \\
& \lambda_{2} & & & \\
& & \cdot & & \\
& & & \lambda_{\lambda_{n+1}}
\end{array}\right), \quad \lambda_{i} \in \boldsymbol{R}, i \in\{1,2, \cdots, n+1\},
$$

(see also [4] for the case of surfaces of revolution $M^{2}$ in $E^{3}$ ). This means that he imposes the condition that the coordinate functions of $M^{n}$ are eigenfunctions of their Laplacian $\Delta$ with possibly distinct eigenvalues $\lambda_{2}$; hence, O. Garay's condition ((1.5), (1.6)) can be seen as a generalization of T. Takahashi's condition (1.2), in which case all $\lambda_{2}$ are equal. O. Garay proved that if a hypersurface $M^{n}$ of $\boldsymbol{E}^{n+1}$ satisfies his condition, it is either minimal in $\boldsymbol{E}^{n+1}$ or it is a sphere or it is a spherical cylinder. In this respect, we want to observe however that his condition is not coordinate-invariant; e.g. in $\boldsymbol{E}^{3}$ a circular cylinder satisfies this condition if and only if its axis of symmetry is one of the coordinate axes.

In this paper, we will study the surfaces in $\boldsymbol{E}^{3}$ which satisfy

$$
\Delta x=A x+B,
$$

where $A \in \boldsymbol{R}^{3 \times 3}$ and $B \in \boldsymbol{R}^{3}$. This setting generalizes T. Takahashi's condition, following O. Garay's idea, in a way which is independent of the choice of coordinates. Our main result is the following.

THEOREM. A surface $M^{2}$ in $\boldsymbol{E}^{3}$ satisfies $\left(^{*}\right)$ if and only if it is an open part of a minimal surface, a sphere or a circular cylinder.

In particular, this yields the following

COROLlary. A compact surface in $\boldsymbol{E}^{3}$ satisfies $\left(^{*}\right)$ if and only if it is a sphere.

We want to mention that this Corollary supports the above Conjecture of B. Y. Chen. Indeed, the compact surfaces $M^{2}$ in $\boldsymbol{E}^{3}$ satisfying $\left(^{*}\right)$ are particular surfaces of finite type $(\leqq 3)$; actually, the following arguments, which will make this clear, also hold more generally for any compact submanifold $M^{n}$ in $\boldsymbol{E}^{m}$ which satisfies a condition of the form $\left(^{*}\right)$. Namely, integrating $\left(^{*}\right)$ over $M^{2}$, and using the divergence theorem, implies that

$$
A x_{0}+B=0 \text {. }
$$

Using this, then $\left(^{*}\right)$ further implies that

$$
\Delta\left(x-x_{0}\right)=A\left(x-x_{0}\right),
$$


and, hence, that

$$
P(\Delta)\left(x-x_{0}\right)=P(A)\left(x-x_{0}\right),
$$

where $P$ is any polynomial in one variable. In particular, choosing for $P$ the characteristic polynomial of $A$, by the Cayley-Hamilton theorem $P(A)=0$, and thus (1.9) shows that

$$
P(\Delta)\left(x-x_{0}\right)=0 .
$$

Finally, Theorem A then asserts that $M^{2}$ is a surface of type $\leqq 3$ in $\boldsymbol{E}^{3}$.

We first show that the surfaces mentioned in the theorem indeed satisfy condition $(*)$.

\section{Examples.}

(1) Minimal surface

In this case we have that the mean curvature is zero, so by (1.1) a minimal surface satisfies $\left(^{*}\right)$ with $A=0$.

(2) Sphere

The sphere $S_{0}^{2}(r)$ with center 0 and radius $r$ satisfies $\left(^{*}\right)$ with

$$
A=\left(\begin{array}{ccc}
\frac{2}{r^{2}} & 0 & 0 \\
0 & \frac{2}{r^{2}} & 0 \\
0 & 0 & \frac{2}{r^{2}}
\end{array}\right) .
$$

Indeed, the sphere has mean curvature $-1 / r$ and $(1 / r) x$ is a unit normal on $S_{0}^{2}(r)$. So by $(1.1)$

$$
\Delta x=\frac{2}{r^{2}} x
$$

(3) Circular cylinder

We consider the cylinder on the circle of radius $r$ with center 0 lying in the $\left\{e_{1}, e_{2}\right\}$-plane. This surface has mean curvature $-1 / 2 r$. A unit normal is given by $(1 / r) \pi(x)$, where $\pi$ is the projection on the $\left\{e_{1}, e_{2}\right\}$-plane. Hence by (1.1)

$$
\Delta x=\frac{1}{r^{2}} \pi(x) .
$$

So this cylinder satisfies $(*)$ with

$$
A=\left(\begin{array}{ccc}
\frac{1}{r^{2}} & 0 & 0 \\
0 & \frac{1}{r^{2}} & 0 \\
0 & 0 & 0
\end{array}\right) .
$$


Proof of the Theorem. We consider two cases.

\section{First case : $M^{2}$ is a cylinder.}

In this case, the position vector $x$ of $M^{2}$ can be given by

$$
x=\gamma(s)+t \xi
$$

where $s, t$ are parameters, $\xi$ is a constant vector and $\gamma(s)$ is a curve, with arclength parametrization, in a plane orthogonal to $\xi$.

From the definition of the Laplacian, one checks that

$$
\Delta x=\gamma^{\prime \prime}
$$

where $\gamma^{\prime \prime}$ is the acceleration vector of $\gamma$.

Without loss of generality we may suppose that $\xi=(0,0,1)$ and that $\gamma(s)=$ $\left(\gamma_{1}(s), \gamma_{2}(s), 0\right)$. If we write

$$
A=\left(\begin{array}{lll}
a_{11} & a_{12} & a_{13} \\
a_{21} & a_{22} & a_{23} \\
a_{31} & a_{32} & a_{33}
\end{array}\right) \text { and } B=\left(\begin{array}{l}
b_{1} \\
b_{2} \\
b_{3}
\end{array}\right) \text {, }
$$

then equation $(*)$ becomes

$$
\begin{gathered}
\gamma_{1}^{\prime \prime}=a_{11} \gamma_{1}+a_{12} \gamma_{2}+a_{13} t+b_{1}, \\
\gamma_{2}^{\prime \prime}=a_{21} \gamma_{1}+a_{22} \gamma_{2}+a_{23} t+b_{2}, \\
0=a_{31} \gamma_{1}+a_{32} \gamma_{2}+a_{33} t+b_{2} .
\end{gathered}
$$

Since $\gamma_{1}^{\prime \prime}, \gamma_{2}^{\prime \prime}$ do not depend on $t$, we find that $a_{13}=a_{23}=a_{33}=0$.

If $a_{31} \neq 0$ or $a_{32} \neq 0$, the curve $\gamma$ is a line, so $M^{2}$ will be part of a plane and hence minimal. So we suppose further that $a_{31}=a_{32}=0$ and that $\gamma$ isn't a line. This implies that $b_{3}=0$. System (2.1) reduces to

or, in vector notation

$$
\begin{aligned}
& \gamma_{1}^{\prime \prime}=a_{11} \gamma_{1}+a_{12} \gamma_{2}+b_{1}, \\
& \gamma_{2}^{\prime \prime}=a_{21} \gamma_{1}+a_{22} \gamma_{2}+b_{2},
\end{aligned}
$$

$$
\gamma^{\prime \prime}=\tilde{A} \gamma+\tilde{B}
$$

where

$$
\tilde{A}=\left(\begin{array}{ll}
a_{11} & a_{12} \\
a_{21} & a_{22}
\end{array}\right) \text { and } \tilde{B}=\left(\begin{array}{l}
b_{1} \\
b_{2}
\end{array}\right) .
$$

We now use the Frenet frame $\{T, N\}$ of the curve $\gamma$. The curve has arc-length 
parametrization, so $T=\gamma^{\prime}$, the velocity vector of $\gamma$.

Equation (2.2) becomes

$$
T^{\prime}=\tilde{A} \gamma+\tilde{B}
$$

Using the Frenet formula $T^{\prime}=\kappa N$ where $\kappa$ is the curvature function of $\gamma$, we get

$$
\kappa N=\tilde{A} \gamma+\tilde{B} .
$$

Derivation of this equation gives

$$
\kappa^{\prime} N+\kappa N^{\prime}=\tilde{A} T .
$$

From the second Frenet formula $N^{\prime}=-\kappa T$ we obtain

$$
\kappa^{\prime} N-\kappa^{2} T=\tilde{A} T \text {. }
$$

We derive again to obtain

or

$$
\kappa^{\prime \prime} N+\kappa^{\prime} N^{\prime}-2 \kappa \kappa^{\prime} T-\kappa^{2} T^{\prime}=\tilde{A} T^{\prime}
$$

$$
\left(\kappa^{\prime \prime}-\kappa^{3}\right) N-3 \kappa \kappa^{\prime} T=\kappa \tilde{A} N .
$$

From (2.3) and (2.4) we can compute the entries of the matrix $\tilde{A}$ with respect to the frame $\{T, N\}$

$$
\begin{aligned}
& \tilde{A} T . T=-\kappa^{2}, \\
& \tilde{A} T . N=\kappa^{\prime}, \\
& \tilde{A} N . T=-3 \kappa^{\prime}, \\
& A N . N=\frac{1}{\kappa}\left(\kappa^{\prime \prime}-\kappa^{3}\right) .
\end{aligned}
$$

The determinant $(\tilde{A} T . T)(\tilde{A} N . N)-(\tilde{A} T . N)(\tilde{A} N . T)$ and the trace $(\tilde{A} T . T)+(\tilde{A} N . N)$ of the matrix $\tilde{A}$ are constant, so there exist constants $c$ and $d$ such that

$$
\begin{gathered}
-\kappa \kappa^{\prime \prime}+\kappa^{4}+3\left(\kappa^{\prime}\right)^{2}=c, \\
\frac{\kappa^{\prime \prime}}{\kappa}-2 \kappa^{2}=d .
\end{gathered}
$$

Eliminating $\kappa^{\prime \prime}$ from these two equations we find that

$$
\left(\kappa^{\prime}\right)^{2}=\frac{1}{3}\left(c+d \kappa^{2}+\kappa^{4}\right) .
$$

Deriving this last equation gives

$$
\kappa^{\prime} \kappa^{\prime \prime}=\frac{1}{3}\left(d \kappa \kappa^{\prime}+2 \kappa^{3} \kappa^{\prime}\right) .
$$


If we suppose that $\kappa^{\prime} \neq 0$, then we have

$$
\kappa^{\prime \prime}=\frac{1}{3}\left(d \kappa+2 \kappa^{3}\right)
$$

Substitution in (2.6) gives

$$
\kappa\left(d+2 \kappa^{2}\right)=0
$$

which contradicts the assumption that $\kappa^{\prime}$ wasn't identically zero. Hence the only solution to the system $(2.2)$ is that $\kappa$ is a constant and that $\gamma$ is a circle. So the only cylinder which satisfies $\left(^{*}\right)$ is a circular cylinder.

\section{Second case : $M^{2}$ is not a cylinder.}

(1) Rank of $A$ is 3 .

In this case we may suppose that $B=0$. Indeed, let $C \in \boldsymbol{R}^{3 \times 1}$ be a solution of $A . C+B=0$. Define new coordinates $x^{\prime}$ by $x=x^{\prime}+C$. Then equation (*) becomes

$$
\Delta x^{\prime}=A x^{\prime} .
$$

Suppose now that $M^{2}$ is given locally as the graph of a function $f$, this is

$$
x=\left(x_{1}, x_{2}, f\left(x_{1}, x_{2}\right)\right) .
$$

From (1.1) we see that $\Delta x=A x$ is normal to the surface, so

$$
A x \cdot\left(1,0, \frac{\partial f}{\partial x_{1}}\right)=0 \text {, }
$$

$$
A x \cdot\left(0,1, \frac{\partial f}{\partial x_{2}}\right)=0,
$$

since $\left(1,0, \partial f / \partial x_{1}\right)$ and $\left(0,1, \partial f / \partial x_{2}\right)$ are tangent vectors.

Let

$$
A=\left(\begin{array}{lll}
a_{11} & a_{12} & a_{13} \\
a_{21} & a_{22} & a_{23} \\
a_{31} & a_{32} & a_{33}
\end{array}\right)
$$

then system (3.1) becomes

$$
\begin{aligned}
& \frac{\partial f}{\partial x_{1}}=-\frac{a_{11} x_{1}+a_{12} x_{2}+a_{13} f}{a_{31} x_{1}+a_{32} x_{2}+a_{33} f}, \\
& \frac{\partial f}{\partial x_{2}}=-\frac{a_{21} x_{1}+a_{22} x_{2}+a_{23} f}{a_{31} x_{1}+a_{32} x_{2}+a_{33} f} .
\end{aligned}
$$

Since the function $f$ satisfies 


$$
\frac{\partial^{2} f}{\partial x_{1} \partial x_{2}}=\frac{\partial^{2} f}{\partial x_{2} \partial x_{1}}
$$

the two above equations imply that

$$
\begin{aligned}
& \left(a_{21}-a_{12}\right)\left(a_{31} x_{1}+a_{32} x_{2}+a_{33} f\right) \\
& \quad+\left(a_{32}-a_{23}\right)\left(a_{11} x_{1}+a_{12} x_{2}+a_{13} f\right) \\
& \quad+\left(a_{13}-a_{31}\right)\left(a_{21} x_{1}+a_{22} x_{2}+a_{23} f\right)=0 .
\end{aligned}
$$

We may suppose that $x_{1}, x_{2}$ and $f$ are linearly independent, and so we get

$$
\begin{aligned}
& \left(a_{21}-a_{12}\right) a_{31}+\left(a_{32}-a_{23}\right) a_{11}+\left(a_{13}-a_{31}\right) a_{21}=0, \\
& \left(a_{21}-a_{12}\right) a_{32}+\left(a_{32}-a_{23}\right) a_{12}+\left(a_{13}-a_{31}\right) a_{22}=0, \\
& \left(a_{21}-a_{12}\right) a_{33}+\left(a_{32}-a_{23}\right) a_{13}+\left(a_{13}-a_{31}\right) a_{23}=0 .
\end{aligned}
$$

If we denote the cofactor of the entry $a_{\imath \jmath}$ in the matrix $A$ by $A_{\imath \jmath}$, this system reduces to

$$
\begin{aligned}
& A_{23}=A_{32}, \\
& A_{13}=A_{31}, \\
& A_{12}=A_{21},
\end{aligned}
$$

i. e. the matrix $A^{\text {cof }}$ of cofactors of $A$ is symmetric. Since

$$
A^{-1}=\frac{1}{\operatorname{det} A} \cdot A^{\text {cof }}
$$

we find that $A^{-1}$ is symmetric. Hence $A$ is also a symmetric matrix.

After a coordinate transformation we may suppose that $A$ is a diagonal matrix

with $\lambda_{1} \cdot \lambda_{2} \cdot \lambda_{3} \neq 0$.

$$
A=\left(\begin{array}{ccc}
\lambda_{1} & 0 & 0 \\
0 & \lambda_{2} & 0 \\
0 & 0 & \lambda_{3}
\end{array}\right)
$$

Suppose now that $\left(x_{1}(u, v), x_{2}(u, v), x_{3}(u, v)\right)$ is a parametrization of the surface. Then, since $A x=\left(\lambda_{1} x_{1}, \lambda_{2} x_{2}, \lambda_{3} x_{3}\right)$ is normal to the surface, we have that

$$
\begin{aligned}
& \left(\lambda_{1} x_{1}, \lambda_{2} x_{2}, \lambda_{3} x_{3}\right) \cdot\left(\frac{\partial x_{1}}{\partial u}, \frac{\partial x_{2}}{\partial u}, \frac{\partial x_{3}}{\partial u}\right)=0, \\
& \left(\lambda_{1} x_{1}, \lambda_{2} x_{2}, \lambda_{3} x_{3}\right) \cdot\left(\frac{\partial x_{1}}{\partial v}, \frac{\partial x_{2}}{\partial v}, \frac{\partial x_{3}}{\partial v}\right)=0,
\end{aligned}
$$


or

$$
\begin{aligned}
& \frac{\partial}{\partial u}\left(\lambda_{1} x_{1}^{2}+\lambda_{2} x_{2}^{2}+\lambda_{3} x_{3}^{2}\right)=0, \\
& \frac{\partial}{\partial v}\left(\lambda_{1} x_{1}^{2}+\lambda_{2} x_{2}^{2}+\lambda_{3} x_{3}^{2}\right)=0 .
\end{aligned}
$$

So

$$
\lambda_{1} x_{1}^{2}+\lambda_{2} x_{2}^{2}+\lambda_{3} x_{3}^{2}=c,
$$

where $c$ is a constant, and we see that $M^{2}$ is part of a quadratic surface. For this quadratic surface one computes the mean curvature

$$
\|H\|= \pm \frac{\left(\lambda_{2}+\lambda_{3}\right) \lambda_{1}^{2} x_{1}^{2}+\left(\lambda_{1}+\lambda_{3}\right) \lambda_{2}^{2} x_{2}^{2}+\left(\lambda_{1}+\lambda_{2}\right) \lambda_{3}^{2} x_{3}^{2}}{2\left(\lambda_{1}^{2} x_{1}^{2}+\lambda_{2}^{2} x_{2}^{2}+\lambda_{3}^{2} x_{3}^{2}\right)^{3 / 2}} .
$$

From $\left(^{*}\right)$ and (1.1), we have that the absolute value of the mean curvature equals $(1 / 2)\|A x\|$, which implies that

$$
\left(\lambda_{1}^{2} x_{1}^{2}+\lambda_{2}^{2} x_{2}^{2}+\lambda_{3}^{2} x_{3}^{2}\right)^{2} \pm\left(\left(\lambda_{2}+\lambda_{3}\right) \lambda_{1}^{2} x_{1}^{2}+\left(\lambda_{1}+\lambda_{3}\right) \lambda_{2}^{2} x_{2}^{2}+\left(\lambda_{1}+\lambda_{2}\right) \lambda_{3}^{2} x_{3}^{2}\right)=0 .
$$

From (3.2) we have that

$$
x_{3}^{2}=\frac{1}{\lambda_{3}}\left(c-\lambda_{1} x_{1}^{2}-\lambda_{2} x_{2}^{2}\right) .
$$

If we substitute this in (3.3), we obtain a polynomial in $x_{1}$ and $x_{2}$ which has to be identically zero, so in particular the coefficients of $x_{1}^{4}$ and $x_{2}^{4}$, which are $\lambda_{1}^{2}\left(\lambda_{1}-\lambda_{3}\right)^{2}$ respectively $\lambda_{2}^{2}\left(\lambda_{2}-\lambda_{3}\right)^{2}$ have to be zero. So we find that $\lambda_{1}=\lambda_{2}=\lambda_{3}$. Hence $M^{2}$ is a sphere. The constant term of the polynomial, which is $c \lambda_{3}\left(c \lambda_{3}-\lambda_{1}-\lambda_{2}\right)$, also has to be zero. From this we find that $c=2$. So if we write $r$ for the radius of the sphere we have

$$
\lambda_{1}=\lambda_{2}=\lambda_{3}=\frac{2}{r^{2}} \text {. }
$$

(2) Rank of $A$ is 2 .

By choosing a basis $\left\{e_{1}, e_{2}, e_{3}\right\}$ with $e_{1}, e_{2} \in \operatorname{Im} A$ and $e_{3} \in(\operatorname{Im} A)^{\perp}$, we may suppose that $A$ and $B$ have the form

$$
A=\left(\begin{array}{ccc}
a_{11} & a_{12} & a_{13} \\
a_{21} & a_{22} & a_{23} \\
0 & 0 & 0
\end{array}\right) \text { and } B=\left(\begin{array}{l}
0 \\
0 \\
b_{3}
\end{array}\right)
$$

If $B=0$, then $\Delta x=-2 H$ belongs to $\operatorname{Im} A$ which is a plane through the origin. This means that the normal on this plane is a constant tangent direction to $M^{2}$, but this isn't possible since $M^{2}$ isn't a cylinder. So we may suppose that $b_{3} \neq 0$.

Consider the set 
Since

$$
U=\left\{p \in M^{2} \mid\left(e_{3}\right)_{p} \notin T_{p} M^{2}\right\} .
$$

$$
U=\left\{\left.p \in M^{2}|| \begin{array}{ll}
\left.\frac{\partial x_{1}}{\partial u}\right|_{p} & \left.\frac{\partial x_{2}}{\partial u}\right|_{p} \\
\left.\frac{\partial x_{1}}{\partial v}\right|_{p} & \left.\frac{\partial x_{2}}{\partial v}\right|_{p}
\end{array}\right|_{\neq 0}\right\},
$$

this is an open set, and by the assumption that $M^{2}$ is not a cylinder, $U$ cannot be empty. By the inverse function theorem, on $U$ the surface is locally given as the graph of a function $f$ in the following way

$$
x=\left(x_{1}, x_{2}, f\left(x_{1}, x_{2}\right)\right) .
$$

From (1.1) we see that $\Delta x=A x+B$ is normal to the surface, so

$$
\begin{aligned}
& (A x+B) \cdot\left(1,0, \frac{\partial f}{\partial x_{1}}\right)=0, \\
& (A x+B) \cdot\left(0,1, \frac{\partial f}{\partial x_{2}}\right)=0,
\end{aligned}
$$

or

$$
\begin{aligned}
& \frac{\partial f}{\partial x_{1}}=\frac{1}{b_{3}}\left(a_{11} x_{1}+a_{12} x_{2}+a_{13} f\right), \\
& \frac{\partial f}{\partial x_{2}}=\frac{1}{b_{3}}\left(a_{21} x_{1}+a_{22} x_{2}+a_{23} f\right) .
\end{aligned}
$$

Since $f$ satisfies

$$
\frac{\partial^{2} f}{\partial x_{1} \partial x_{2}}=\frac{\partial^{2} f}{\partial x_{2} \partial x_{1}}
$$

we have

$$
\left(a_{12}-a_{21}\right) b_{3}+\left(a_{13} a_{21}-a_{11} a_{23}\right) x_{1}+\left(a_{13} a_{22}-a_{12} a_{23}\right) x_{2}=0,
$$

or

$$
\begin{gathered}
\left|\begin{array}{ll}
a_{11} & a_{13} \\
a_{21} & a_{23}
\end{array}\right|=0, \\
\left|\begin{array}{ll}
a_{12} & a_{13} \\
a_{22} & a_{23}
\end{array}\right|=0, \\
a_{12}=a_{21} .
\end{gathered}
$$

Since $A$ has rank 2, expressions (3.4) and (3.5) imply that

$$
a_{13}=a_{23}=0 \text {. }
$$


Equation (3.6) shows that the matrix $\left(\begin{array}{ll}a_{11} & a_{12} \\ a_{21} & a_{22}\end{array}\right)$ is symmetric. By a coordinate transformation we may suppose that $A$ has the form

with $\lambda_{1} \cdot \lambda_{2} \neq 0$.

$$
\left(\begin{array}{ccc}
\lambda_{1} & 0 & 0 \\
0 & \lambda_{2} & 0 \\
0 & 0 & 0
\end{array}\right)
$$

Suppose now that $\left(x_{1}(u, v), x_{2}(u, v), x_{3}(u, v)\right)$ is a parametrization of the surface. Then, since $A x+B=\left(\lambda_{1} x_{1}, \lambda_{2} x_{2}, b_{3}\right)$ is normal to the surface, we have that

$$
\begin{aligned}
& \left(\lambda_{1} x_{1}, \lambda_{2} x_{2}, b_{3}\right) \cdot\left(\frac{\partial x_{1}}{\partial u}, \frac{\partial x_{2}}{\partial u}, \frac{\partial x_{3}}{\partial u}\right)=0, \\
& \left(\lambda_{1} x_{1}, \lambda_{2} x_{2}, b_{3}\right) \cdot\left(\frac{\partial x_{1}}{\partial v}, \frac{\partial x_{2}}{\partial v}, \frac{\partial x_{3}}{\partial v}\right)=0,
\end{aligned}
$$

or

$$
\begin{aligned}
& \frac{\partial}{\partial u}\left(\lambda_{1} x_{1}^{2}+\lambda_{2} x_{2}^{2}+2 b_{3} x_{3}\right)=0, \\
& \frac{\partial}{\partial v}\left(\lambda_{1} x_{1}^{2}+\lambda_{2} x_{2}^{2}+2 b_{3} x_{3}\right)=0 .
\end{aligned}
$$

So

$$
\lambda_{1} x_{1}^{2}+\lambda_{2} x_{2}^{2}+2 b_{3} x_{3}=c,
$$

where $c$ is a constant, and we see that $M^{2}$ should be part of a quadratic surface. However, for this quadratic surface one computes the mean curvature

$$
\|H\|= \pm \frac{\lambda_{1}^{2} \lambda_{2} x_{1}^{2}+\lambda_{1} \lambda_{2}^{2} x_{2}^{2}+\left(\lambda_{1}+\lambda_{2}\right) b_{3}^{2}}{2\left(\lambda_{1}^{2} x_{1}^{2}+\lambda_{2}^{2} x_{2}^{2}+b_{3}^{2}\right)^{3 / 2}} .
$$

The absolute value of the mean curvature equals $(1 / 2)\|A x+B\|$ by (1.1). This implies that the polynomial

$$
\left(\lambda_{1}^{2} x_{1}^{2}+\lambda_{2}^{2} x_{2}^{2}+b_{3}^{2}\right)^{2} \pm\left(\lambda_{1}^{2} \lambda_{2} x_{1}^{2}+\lambda_{1} \lambda_{2}^{2} x_{2}^{2}+\left(\lambda_{1}+\lambda_{2}\right) b_{3}^{2}\right)
$$

should be identically zero, which contradicts $\lambda_{1} \cdot \lambda_{2} \neq 0$.

(3) Rank of $A$ is 1 .

Since $\Delta x=-2 H$, equation $\left(^{*}\right)$ implies that $-2 H$ lies on $\operatorname{Im} A+B$ which is a line. So a vector orthogonal to a plane which contains the line $\operatorname{Im} A+B$ and the origin, is everywhere tangent to the surface $M^{2}$. This contradicts our assumption that $M^{2}$ isn't a cylinder. 
(4) Rank of $A$ is 0 .

In this case $\left(^{*}\right)$ becomes

$$
\Delta x=B .
$$

If $B=0$, then we have by (1.1) that $H=0$, so the surface is minimal. If $B \neq 0$, equation (1.1) implies that $B$ is a constant vector normal to $M^{2}$, so $M^{2}$ is a plane. However for a plane we have that $H=0$, which contradicts $B \neq 0$.

\section{REFERENCES}

[1] B. Y. Chen, On the total curvature of immersed manifolds, VI: Submanifolds of finite type and their applications, Bull. Inst. Math. Acad. Sinica 11 (1983), 309328.

[2] B.Y. CHEN, "Total mean curvature and submanifolds of finite type," World Scientific, Singapore, 1984.

[3] B. Y. CHEN, Surfaces of finite type in Euclidean 3-space, Bull. Soc. Math. Belg. Sér. B 39 (1987), 243-254.

[4] O. GARAY, On a certain class of finite type surfaces of revolution, Kodai Math. J. 11 (1988), 25-31.

[5] O. Garay, An extension of Takahashi's theorem, preprint.

[6] T. TAKAhashi, Minimal immersions of Riemannian manifolds, J. Math. Soc. Japan 18 (1966), 380-385.

1980 Mathematics subject classifications : 53A05

WisKUNDIG INSTITUUT,

Katholieke Universiteit LeUVEN,

Celestijnenl.aAn 200-B, 3030 Heverlee,

BELGIUM 